\title{
MAGNA MATER IN WANDELNDEM WIRKUNGSKREIS
}

\begin{abstract}
Summary: In the poetry of the late Republic and the age of Augustus, a gradual expansion and Romanisation of the role of Magna Mater can be observed. In Catullus, she appears only as a goddess from Asia Minor linked to Attis' repulsive act. In Lucretius, Cybele is identical with Rhea, which remarkably changes her position as she becomes the mother of all Olympian gods. In Vergil, in addition to being the mother of all gods, she is also the Chief Goddess of the Trojans, who plays an active role in shaping Aeneas' fate. The most thorough picture of the Goddess is provided by Ovid, who covers every detail of the cult, placing emphasis on embracing the cult. He goes to great lengths to attribute Roman origin to its apparently foreign features, i.e., he tries to Romanise the already embraced cult as much as possible. All this must have taken place under the aegis of and in accordance with Augustus' religious policy.
\end{abstract}

Key words: Magna Mater, Attis, Romanisation of the cult, religiouspolicy of Augustus

Zur Rezeption des Kultes der Magna Mater kommt es in Rom im Laufe der Jahre 205-204 v. Chr. Annähernd zum Ende des zweiten punischen Krieges schlugen die sibyllinischen Bücher die Rezeption nicht eines griechischen, sondern eines phrygischen Kultes vor, und zwar des Kultes der kleinasiatischen Göttermutter. Der heilige Stein der Göttin wurde unter Mitwirkung der römischen Adelsfamilien im Tempel der Victoria auf dem Palatin untergebracht, dann wurde 191 v. Chr. der neue Tempel in der unmittelbaren Nachbarschaft aufgebaut. Der Tempel stand also innerhalb des Pomerium, im Zentrum Roms, solange die Pflege des Kultes von XVviri versehen wurde. Schon 194 v. Chr. haben szenische Spiele zu ihrer Ehre stattgefunden. ${ }^{1}$ Zur Einweihung des Tempels wurden die ständigen Festspiele, die Ludi Megalenses, gegründet, die zwischen dem 4. und dem 10. April veranstaltet wurden.

Trotz alledem blieb der Kult in Rom überraschend isoliert. Den römischen Bürgern war es verboten, in seine priesterliche Körperschaft einzutreten und eine Selbstverstümmelung zu begehen. Nur an bestimmten Tagen des Jahres durften die sog.

\footnotetext{
${ }^{1}$ WissowA, G.: Religion und Kultus der Römer [Hb. der Altertumswiss.]. München 1912, 317 ff.
} 
galli mit wilder Musik, in ungewohnter Kleidung durch die Straßen Roms ziehen und um Almosen betteln. Ihre kultische Tätigkeit beschränkte sich praktisch auf das Gebiet des Tempels. ${ }^{2}$ Das Eintreten in das priesterliche Kollegium war nicht nur den römischen Bürgern verboten. Ein Sklave, der in die Reihe der galli eingetreten ist, wurde für immer aus Rom ausgewiesen. ${ }^{3}$ Diese Widersprüchlichkeit und Vielfarbigkeit der sozialen Lage des Kultes spiegelt sich auch in der römischen Dichtkunst der spätrepublikanischen und augusteischen Zeit wider.

In dem Gedicht 63 von Catull erscheint nur die den Römern fremde Seite des Kultes. Der zufälligerweise Attis genannte griechische Bürger fährt an den phrygischen Kultort der Magna Mater und entmannt sich dort unter der Wirkung des heiligen Wahnsinnes (5-6: stimulatus ibi furenti rabie ... devolsit ili acuto sibi pondera silice). In dem Hain der Göttin empfangen ein Zinnteller (21: cymbalum), eine Trommel (21: tympanon) und eine krumme Flöte (22: curvus calamus) die in Verzückung geratenen Gläubigen. Am folgenden Tag, als die Verzückung beendet ist (44: sine rabie; 57: rabie fera carens), wird sich Attis seiner fürchterlichen Tat und seines Unheils bewusst (61: miser a miser). Da gereut es ihn schon, was er in der Ekstase begangen hat: iam iam dolet quod egi, iam iamque paenitet (73). Die Göttin lässt aber ihren Anhänger nicht mit dem Zweifel ringen, sondern sorgt mit Hilfe eines ihrer Löwen dafür, dass der Wahnsinn seiner wieder Herr wird und er in ihren heiligen Hain flüchtet, wo er der Göttin bis zum Ende seines Lebens dient: fac ut hunc furor agitet, fac uti furoris ictu reditum in nemora ferat (78-9). In den Endzeilen des Gedichtes bittet Catull die große Göttin (91: magna dea) darum, dass sein Haus von diesem Wahnsinn verschont bleibt (92: procul a mea ... sit furor ... domo) und andere ihm zum Opfer fallen (93: alios age incitatos, alios age rabidos). Der Dichter streicht also die widerlichste Seite des Kultes heraus, die er vollständig zurückweist. Was würde mit ihm geschehen, wenn er gerade den Früchten der Liebe entsagen müsste.

Ein vollständig anderes Bild zeichnet sich in der Beschreibung der Magna Mater bei Lukrez (2. 598-643) ab. Hier erscheint sie nämlich nicht nur als eine phrygische Göttin, sondern wird mit der griechischen Rhea identifiziert, mit der Mutter aller Lebewesen (598-599: magna deum mater materque ferarum / et nostri genetrix haec dicta est corporis una.). Es bleiben noch die phrygischen Züge der Göttin, galli begleiten sie unterwegs, aber nur um anzuzeigen, was für Konsequenzen die Verletzung der Treue zu ihr hat (614-617: numen qui violarint matris...). Es dröhnen auch die fürchterlichen Musikinstrumente tympana, cymbala, cornua (618-619) in ihrem Gefolge, aber nicht um die Teilnehmer des Prozesses in Ekstase zu versetzen, sondern um in den fehlbaren und sündigen Seelen Respekt vor der Göttin zu erwecken (622623: ingratos animos atque impia pectora volgi conterrere metu...). Sie wird von den wild tanzenden und lärmenden Cureten begleitet, aber nur damit diese das Weinen des kindlichen Jupiter vor Saturn verhehlen (629-639) oder damit sie vorführen, dass die Jünglinge die Aufgabe haben, die Heimat und die Eltern zu verteidigen (641643). Die wilden Sitten des Kultes der Göttin verschwinden also nicht, werden aber

\footnotetext{
2 TAKÁCS, S. A.: Kybele. Der Neue Pauly 6. Stuttgart-Weimar 1999, 953.

${ }^{3}$ LATTE, K.: Römische Religionsgeschichte [Hb. der Altertumswiss.]. München 1960, 260.
} 
nach der griechisch-römischen Gesinnung umgewertet und füllen sich mit ethischem Gehalt.

Dazu kommen noch philosophische Züge. Magna Mater ist nicht nur die Mutter aller Lebewesen, sondern sie ist die Erde selbst oder mindestens deren Symbol, wenn sie mit dem Löwengespann über den Lufthimmel fährt: aeris in spatio magnam pendere docentes tellurem (602-603). Die wilden Löwen symbolisieren die kindliche Wildheit, die von der elterlichen Erziehung besänftigt wird (604-605). Als Erdmutter ist sie die Urmutter des Ackerbaus: edunt per terrarum orbem fruges coepisse creari (612-613) und gleichzeitig die Trägerin der Städte, was die Mauerkrone auf ihrem Kopf symbolisiert: muralique caput summum cinxere corona, / eximiis munita locis quia sustinet urbes (606-607). Kein Wunder, dass die Göttin den Sterblichen (6245 ), die den Weg zum Dank mit Blumen und mit allen Kostbarkeiten bestreuen, Segen bringt, wo sie stumm vorübergeht (626-628).

Ein wiederum anderes Bild der Magna Mater liegt in Vergils Aeneis vor. Im Strom der epischen Geschichte erscheint sie mehrmals, wenn sie stetig das Schicksal der fliehenden Trojaner verfolgt und ihnen, wenn nötig, zur Hilfe eilt. Die Erklärung ihrer Sorge besteht darin, dass Troja auf dem Land von Phrygien liegt und Magna Mater die Hauptgöttin dieses Gebietes ist. Ihren ersten Eingriff sehen wir, als Creusa verschwindet. Der in das zugrunde gehende Troja zurückkehrende Aeneas trifft nur den Geist seiner Gemahlin, die ihn beruhigt, dass ihr Verschwinden der Sorge der Magna Mater zu verdanken ist: sed me magna deum genetrix his detinet oris (2. 788). Schon ihre Benennung verweist darauf, dass es sich um die mit der griechischen Rhea identifizierte Göttin handelt. Das Zurückhalten an den Küsten von Troja bedeutet aller Wahrscheinlichkeit nach den Tod der Creusa. Magna Mater erklärt ihre Tat damit, dass Creusa als Nachfolgerin des Dardanus und als Schwiegertochter der Venus nicht in die Hände der Griechen fallen darf, wo sie zur Sklavin gemacht würde. Im Spiegel der späteren Geschehnisse der Aeneis ist aber offensichtlich, dass bei den Vorfällen auch eine andere Überlegung eine Rolle spielen konnte. Nämlich wäre Creusa ein Hindernis für das Zustandekommen des Bundes zwischen Aeneas und Lavinia in Latium gewesen.

Der zweite Eingriff der Magna Mater in den Strom der Geschehnisse erfolgt in dem 9. Gesang, also auf dem Land Italiens. In Folge des Vorstoßes der Rutuler bedroht ein Feuerbrand die Schiffe der Trojaner. Die Schiffe wurden aber nicht aus irgendeinem Holz, sondern aus dem Nadelholz des heiligen Haines der Cybele auf dem Berg Ida verfertigt (9. 85-89). Deshalb wendet sich die Göttin an ihren Sohn Jupiter, damit diese Schiffe nicht vernichtet werden (9. 90-92). Jupiter verspricht, dass er sie in unsterbliche Meeresgötter verwandeln werde: mortalem eripiam formam magnique iubebo / aequoris esse deas (9. 101-102). Die in Meeresnymphen verwandelten Schiffe bekommen eine Rolle im 10. Gesang. Sie suchen Aeneas auf, der unterwegs ist, und stellen sich vor: Nos sumus Idaeae sacro de vertice pinus, / nunc pelagi nymphae, classis tua. (10. 230-231) ... Hanc genetrix faciem miserata refecit... (10. 234). Sie machen ihn darauf aufmerksam, dass sein Lager in Gefahr ist und er ehestens dahin zurückkehren soll (10. 228-245). Gleichzeitig beschleunigen sie die Fahrt seines Schiffes. Aeneas empfindet, dass es sich um einen göttlichen Eingriff 
handelt und fleht Magna Mater (252: Alma parens Idaea deum) an, dass sie bei der Beschützung des Lagers helfen solle (10. 254-255).

Die Göttin erscheint in dem Text der Aeneis nicht nur unmittelbar, sondern auch mittelbar. Unter diesen Stellen ist die bedeutendste ein Gleichnis, mit dem Vergil im 6. Gesang Roms zukünftige Größe ausmalt (781-787). Das mit dem Erdkreis gleichzusetzende Rom wird so mit männlichen Nachkommen gesegnet (784: felix prole virum), wie die durch die phrygischen Städte reisende Berecynthia mater mit göttlichen Nachkommen reich gesegnet ist (786: laeta deum partu), die das himmlische Reich besitzen (787).

In den erwähnten Beispielen fällt auf, dass die Magna Mater bei Vergil eine ausgezeichnete Rolle spielt. Diese Erscheinung hat T. P. Wiseman ${ }^{4}$ untersucht und eine Erklärung dafür gesucht. Er stellte die Frage, warum die Göttin für Augustus so wichtig war - und deshalb auch für Vergil -, dass er den Platz seines Hauses auf dem Palatin in Nachbarschaft des Tempels der Magna Mater gewählt hat. ${ }^{5}$ Die Erklärung sieht er darin, dass Augustus sich mit den Symbolen des Sieges und der Gründung Roms umgeben wollte. ${ }^{6}$ In seiner Nachbarschaft waren der Tempel von Victoria, der mit Actium zusammenhängende Tempel von Apollo, das Lupercal, die Hütte des Romulus und die Scalae Caci. In diese Reihe gehörte auch die Magna Mater, die bei Vergil zur Gottheit der Trojaner geworden ist, und der Berg Ida verkörperte nicht mehr den Platz des catullischen Entsetzens, sondern den Wohnort der Göttin, die die Sicherheit der ihre Heimat suchenden Urahnen gewährleistete.

Das vollständigste Bild der Magna Mater liegt bei Ovid vor, der in den Fasten bei der Beschreibung der Feierlichkeit der Megalesia fast 200 Zeilen der Vorstellung des Ursprungs des Ereignisses widmet (4. 179-372). Auch bei ihm ist die auffälligste Erscheinung - wie bei Lukrez - die laute Musik, die die tibia, die tympana und die cymbala erwecken; sowie die semimares, d.h. die galli. Der Dichter stellt Fragen in Verbindung mit dem Gesehenen, bekommt aber die Antwort nicht von der Göttin, sondern von den gelehrten Enkeln (doctae nepotes), von den Musen. Die genealogische Verbindung weist darauf hin, dass Cybele auch hier, wie bei Lukrez und Vergil, mit der griechischen Rhea, der Mutter der Götter, identisch ist. Das Attribut doctae weist zugleich darauf hin, dass wir mit fachgemäßen Erklärungen der ungewohnten Erscheinungen rechnen können. Bis Lukrez gibt es eigene Erklärungen für die laute Musik und das Gelärm der Cureten und er verknüpft nur Letzteres mit Jupiters Geburt, während bei Ovid die lauten Musikinstrumente die lärmigen Instrumenten der Cureten vertreten: Cymbala pro galeis, pro scutis tympana pulsant; / tibia dat Phrygios, ut dedit ante modos (4. 213-214). Der Gebrauch der lauten Musikinstrumente bekommt also eine aetiologische Erklärung, während er bei Lukrez zur seelischen Vorbereitung der Gläubigen, zur Erweckung des Respekts vor der Göttin gehört. Das ins-Joch-Spannen der Löwen bedeutet das Bezähmen der Wildheit (4. 215-218) und

${ }^{4}$ Wiseman, T. P.: Cybele, Virgil and Augustus. In Poetry and Politics in the Age of Augustus. Ed. T. WoOdman - D. West. Cambridge 1984, 117-128 = Augustus. Ed. J. EdmOndson. Edinburgh 2009, 381-398.

\footnotetext{
${ }^{5}$ WiSEMAN (Anm. 4) 125.

${ }^{6}$ WisEMAN (Anm. 4) $126 \mathrm{f}$.
} 
beschränkt sich nicht auf die kindliche Wildheit. Die Mauerkrone auf dem Kopf der Göttin weist bei Lukrez auf die Städte hin, die die Erde trägt, bei Ovid dagegen bedeutet sie, dass sie den Städten zuerst Türme gab (4. 219-220).

Die nächste, lang ausgeführte Frage ist die Erklärung der Selbstverstümmelung (4. 221-246). Lukrez erledigt das sehr kurz: Sie war die Konsequenz der Beleidigung der Göttermutter. Dagegen erzählt Ovid die Geschichte von Attis, der gegen die Erwartung der Göttin und gegen das eigene Versprechen verstoßend ein so großes Schuldbewusstsein fühlte, dass ihn Raserei ergriff (233: furit) und er sich um den Körperteil brachte, womit er die Sünde begangen hatte. Diesem Beispiel folgend und in ähnlicher Raserei (243, 246: furor) begehen ihre unmännlichen Priester (243: $\mathrm{mol}$ les ministri) die Selbstverstümmelung. Eine weitere, lang ausgeführte Frage ist der Ursprung der Göttin und ihre Ankunft in Rom (4. 247-349). Mit diesen Fragen beschäftigt sich Lukrez überhaupt nicht, bei Vergil bekommt aber der Ursprung des Kultes eine entscheidende Rolle. Dafür gibt es auch einen textlichen Hinweis: Als Aeneas Troja nach Italien brachte, wollte die Göttin selbst ihm nachfolgen: cum Troiam Aeneas Italos portaret in agros, / est dea sacriferas paene secuta rates (251-252). Unter Troja ist vor allem die beschützenden Götter der Stadt zu verstehen, die Aeneas mitgebracht hat, worauf das Attribut sacrifera neben der ratis hinweist. Wie aus der Aeneis hervorgeht, erfüllte auch die Magna Mater eine ähnliche beschützende Rolle in Phrygien, wo Troja liegt. Die Göttin aber erwartete, dass das Schicksal die Notwendigkeit des Ankommens in Italien kundgebe (257: carminis Euboici fatalia verba). Die wortkarge Prophezeiung vermisst die Mutter (259: Mater abest), bis die Wahrsagung von Apollo präzisiert wird: Es handelt sich um die Mutter der Götter, die im Gebirge Ida aufzufinden ist (263-264: Divum ... arcessite Matrem ... in Idaeo est invenienda iugo). Nachdem die Römer der Göttin Gesandte nach Phrygien geschickt hatten und Attalus nicht zustimmen wollte, geschieht ein Wunder: Im Heiligtum erhebt Cybele die Stimme und äußert ihren Willen, nach Rom zu fahren. Attalus widerspricht nicht mehr, ja, „du bleibst unser“, sagt er, denn auch die Urahnen der Römer waren ursprünglich Phryger: nostra eris: in Phrygios Roma refertur avos. (272). Zum Bau des Schiffes wurden die Kiefern aus demselben Wald geschnitten, aus welchem einst der aus Troja flüchtende Aeneas sie geschnitten hatte, der hier absichtlich Phryge und nicht als Aeneas genannt wird: caedunt pineta secures / illa, quibus fugiens Phryx pius usus erat (273-274).

Detailliert beschreibt Ovid die Meeresroute des die Göttin befördernden Schiffes (277-290), dann ihren Empfang an der Tibermündung (291-296). Da ist die ganze römische Gesellschaft: der Senat, das römische Volk, Mütter, Töchter und die Vestalinnen. Am detailliertesten wird die Fahrt stromaufwärts beschrieben (297-349). Der Wasserstand des Flusses ist nämlich niedrig, das Schiff fährt auf. Jeder Versuch zum Schleppen des Schiffes erweist sich als vergeblich, bis Claudia Quinta, die vornehmer Herkunft ist, deren Keuschheit aber vom Klatsch bezweifelt wurde, das Schleppen übernimmt. Zuerst betet sie aber zu der Göttin und bittet: Wenn sie sie mit reinen Händen zieht, soll sie als die Verkörperung der Keuschheit folgen (324: castas casta sequere manus). Das Wunder geschieht: Das Schiff setzt sich in Bewegung. Die Göttin erscheint also als Verteidigerin der Keuschheit, die in den Augen der Römer ein 
sehr wichtiger moralischer Wert ist. Ein Hinweis auf diese Geschichte kommt früher nur bei Livius vor, der unter den Matronen, die die Göttin empfangen, dem Namen nach Claudia Quinta erwähnt, deren Keuschheit bezweifelt wurde - aber das Schleppen des Schiffes erzählt er nicht (Liv. 29. 14. 9). Das Schiff macht bei der Mündung des Almo halt, und dort kommt es zuerst zum Waschen der Göttin und ihrer heiligen Gegenstände (339-340: ... sacerdos / Almonis dominam sacraque lavit aquis). Diese Zeremonie wurde später jährlich gehalten, spätestens seit der augusteischen Zeit. ${ }^{7}$ In Rom empfing Scipio Nasica die Göttin. Nach dem Rat des delphischen Orakels musste das der untadelhafteste Mann (vir optimus) erledigen. Die Wahl traf auf P. Scipio Nasica (Liv. 29. 14. 8: iudicaverunt in tota civitate virum bonorum optimum esse). Damit liegt wieder ein wichtiger moralischer Wert vor, der sich mit der Göttin verband.

Im Weiteren kommt es noch zu mehreren Fragen und kürzeren Antworten im Zusammenhang mit den merkwürdigen Gewohnheiten des Kultes. Bei diesen Antworten ist die Absicht der Romanisierung auffällig. Das Sammeln des Almosens der Priester, das kleinasiatischen Ursprungs sein kann, bekommt hier die Erklärung, dass Metellus den Tempel der Göttin mit Hilfe von Geldgaben neu gebaut habe und daher diese Gewohnheit geblieben sei (350-352). Ebenso eine römische Gewohnheit ist die gegenseitige Bewirtung an den Festtagen, die das Andenken an die Ortsveränderung der Göttin bewahrt (353-356). Die Identifizierung mit Rhea wird auch dadurch verstärkt, dass sie unter den Feiertagen des Jahres den ersten hat, an dem zirzensische und szenische Spiele veranstaltet wurden (Megalesia ludi), da sie die Muttergöttin ist und ihr die erste Stelle gebührt (357-360). Ein den Römern fremder Zug erscheint nur bei der Erklärung der Benennung von galli (361-365). Deren Ursprung ist auf den kleinasiatischen Fluss Gallus zurückzuführen; wer dessen Wasser trinkt, beginnt zu rasen. Deshalb soll sich jeder davon zurückhalten, der nüchtern bleiben will, und dann wiederholt Ovid die Warnung: qui bibit inde, furit. Ebenso ist das Verzehren des moretum an diesem Feiertag eine römische Gewohnheit, da Gemüse und Käse zu den ältesten Lebensmitteln der Menschheit gehören (367-372).

Aus dem Erzählten wird offensichtlich, dass der Kult der Magna Mater in der augusteischen Dichtung besondere Aufmerksamkeit bekommen hat, und auch andere Zeichen verweisen darauf, dass die Göttin in der augusteischen Religionspolitik eine wichtige Rolle spielte. ${ }^{8}$ Nach der Feststellung von Ilse Becher: „Die augusteischen Dichter ließen bei der Darstellung der Göttermutter unterschiedliche Facetten aufleuchten ${ }^{\prime 9}$ und spiegeln ,in Fragen von Religion und Kult die Wirklichkeit meist nur partiell“". ${ }^{10}$

Zwischen den dichterischen Darstellungen lassen sich aber nicht nur Unterschiede feststellen, sondern auch eine entschiedene Entwicklungslinie, die eine graduelle Erweiterung und eine graduelle Romanisierung des Wirkungskreises der Magna Mater bedeutet. Solange sie bloß als eine kleinasiatische Göttin in Verbindung mit der

\footnotetext{
${ }^{7}$ TAKÁCS (Anm. 2) 954.

${ }^{8}$ BECHER, I.: Der Kult der Mater Magna in augusteischer Zeit. Klio 73 (1991) 157-170.

${ }^{9}$ BeCHER (Anm. 8) 166.

${ }^{10}$ BECHER (Anm. 8) 170.
} 
widerlichen Tat der Geschichte von Attis erscheint, wie bei Catull, lehnt der Dichter den furor mit Recht ab, der nach der Darstellung das Wesen des Kultes bildet. Bei Lukrez ist Cybele mit Rhea identisch, so verändert sich wesentlich ihre Position, denn sie wird die Mutter aller olympischen Götter. Die Geschichte von Attis bleibt nur ein mythisches Beispiel dafür, mit welcher Konsequenz Treubrüchigkeit verbunden ist. Bei Vergil wird Magna Mater neben ihrer Rolle als Göttermutter die Hauptgöttin der Trojaner, die an der Gestaltung des Schicksals des flüchtenden Aeneas tatkräftig teilnimmt. Die Geschichte von Attis spielt hier keine Rolle. Die vollständigste Darstellung über die Göttin gibt zweifellos Ovid. Er kommt auf alle Details des Kultes zu sprechen, so auch auf die Geschichte von Attis, in der auch er das exemplum des furor sieht, der sich aber in der Gegenwart nur auf die unmännlichen Priester beschränke. Die Betonung liegt bei ihm auf der Rezeption des Kultes und er tut alles, seine fremden Zügen einem römischen Ursprung zuzuschreiben, d.h. den rezipierten Kult so weit wie möglich zu romanisieren. „Auf diese Weise war die phrygische Göttin ... dabei, aus einer Asiatin zu einer Römerin zu werden." ${ }^{\text {"11 }}$ Dies alles geschieht wohl unter dem Einfluss der augusteischen Religionspolitik und steht mit ihr im Einklang.

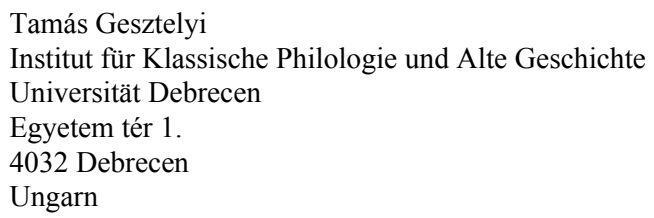

${ }^{11}$ BÖMER, F.: Kybele in Rom. Die Geschichte ihres Kultes als politisches Phänomen. Römische Mitteilungen 71 (1964) 144. 